\title{
Finger Pinch Force Estimation Through Muscle Activations Using A Surface EMG Sleeve On the Forearm
}

\author{
Yinfeng Fang, Zhaojie Ju, Xiangyang Zhu and Honghai Liu
}

\begin{abstract}
For prosthetic hand manipulation, the surface Electromyography(sEMG) has been widely applied. Researchers usually focus on the recognition of hand grasps or gestures, but ignore the hand force, which is equally important for robotic hand control. Therefore, this paper concentrates on the methods of finger forces estimation based on multichannel sEMG signal. A custom-made sEMG sleeve system omitting the stage of muscle positioning is utilised to capture the sEMG signal on the forearm. A mathematic model for muscle activation extraction is established to describe the relationship between finger pinch forces and sEMG signal, where the genetic algorithm is employed to optimise the coefficients. The results of experiments in this paper shows three main contributions: 1) There is a systematical relationship between muscle activations and the pinch finger forces. 2) To estimate the finger force, muscle precise positioning for electrodes placement is not inevitable. 3) In a multi-channel EMG system, selecting specific combinations of several channels can improve the estimation accuracy for specific gestures.
\end{abstract}

\section{INTRODUCTION}

$\mathbf{T}$ HE surface EMG signal is widely used to recognise human motions[1], [2], [3], [4] and estimate muscular force[5]. Plenty of achievements have been made in their respective fields. There is, however, a big gap between these two issues. Researchers who focus on the hand force usually do not care about the gesture recognition, vice versa. In the field of myo-prosthetic hands manipulation, it is equally important to analyse the hand moving trajectories and also to study the magnitude of the force[6], [7]. A decent example is picking up an egg using an sEMG controlled prosthetic hand for amputees, which is definitely a big challenge, especially in an open-loop control system (vision feedback excluded). This paper will focus on the finger force estimation from a 16-channel sEMG sleeve worn on the forearm.

Determining muscle forces through sEMG usually requires a model of muscle contraction dynamics. Huxley-type models that estimate the forces in cross-bridges are very complex and the muscle dynamics are governed by multiple differential equations, which need to be numerically integrated and time-consuming in multiple muscles. Therefore, many

Yinfeng Fang, Zhaojie Ju, and Honghai Liu are with Intelligent Systems and Biomedical Robotics Group, School of Computing, University of Portsmouth, Portsmouth, PO1 3HE (email: \{Yinfeng.Fang, Zhaojie.Ju, Honghai.Liu\}@port.ac.uk).

Xiangyang Zhu is with the State Key Laboratory of Mechanical System and Vibration, Shanghai Jiao Tong University, Shanghai, P.R. China (email:mexyzhu@sjtu.edu.cn)

This work was supported by the project of State Key Lab of Digital Manufacturing Equipment Technology of China (grant No. DMETKF2013001), the National Basic Research Program (973 Program) of China (Grant No. 2011CB013305) and the UK Engineering and Physical Science Research Council (Grant No. EP/G041377/1)

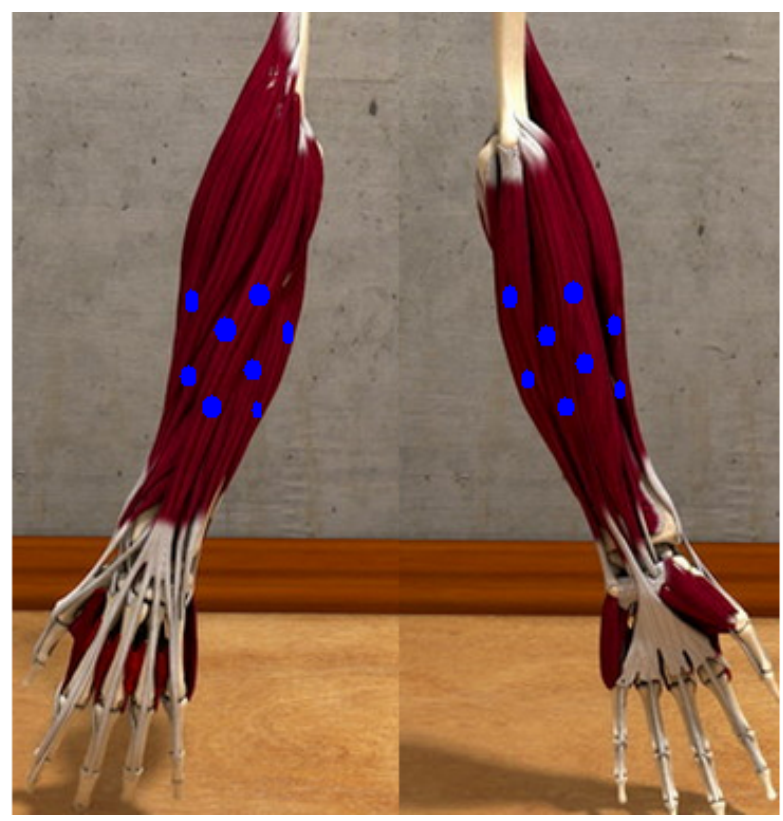

Fig. 1. The electrodes configeration on the forearm

researchers who do large-scale neuromuscular modelling use Hill-type models[8], which is a phenomenological model based on experimental facts with no link to the microscopic physiology[9]. Model-based estimation of muscle forces usually needs optimisation selected to solve for the equations describing the musculoskeletal system [5]. Muscle excitations are iteratively updated by an optimisation algorithm to minimise the tracking error (measured external forces needed) between experimental data and model predictions and satisfy additional constraints[5].

It is not a ultimate goal but an intermediate process for any muscluar dynamics model to estimate the muscular force. Muscluar dynamics models are usually exploited as an investigative tool to discriminate between biomechanical and neural causes of musculoskeletal disorders or diseases affecting the nervous system[10]. In the applications of prosthetic control, however, musculoskeletal parameters are not necessary to be obtained. Leaving out the process of musculoskeletal parameter estimation can reduce the computational consumption. More importantly, model-based force estimation need to pinpoint the specific muscles, which impede its applications beyond the field of disease diagnosis.

Finger related force prediction is difficult due to the large amount of muscles on the forearm and the complex hand structure with more than 20 degrees of freedom. Early in 
1995, Maier, etc. [11], [12], [13] started to explore which muscles or the combination of muscles (muscular synergies) is more suitable for finger force estimation than others during force production in precision grips. Choia, etc., [14] utilise artificial neural network (ANN) to map the SEMG collected through six pieces of muscle (ED, APL, FDS, APB, AP) to the forces. Martin, etc., [15] evaluated the activity pattern of finger flexor muscle and finger force exertion and the relationship between keyboard reaction forces and EMGs while keying. It is suggested that the difference between EMG and finger force partly results from the amount of muscle load not captured by the measured applied force[15]. The existence of enslaving effects [16] in finger force production also increase the hardness in finger force estimation. The experiment of Zatsiorsky, etc., [17] shows that no direct correspondence exists between neural command to an individual finger and the finger force.

This paper will utilise a 16 channel sEMG system to capture the sEMG signals on the forearm[18]. It is named SEMG Sleeve in this paper. All the electrodes are installed in an elastic sleeve with a fixed configuration in advance. This paper is expected to address the following questions. Firstly, whether it is possible to estimate the force dynamic just through processing sEMG signals without considering any muscular dynamic model. Secondly, whether it is possible to estimate the force through multi-channel SEMG Sleeve (no muscle positioning process included). Thirdly, how to make the best of the redundant information in multi-channel SEMG Sleeve to benefit force estimation.

The rest of this paper is organised as follows: Section II states the materials and methods for sEMG signal acquisition and the approach for force estimation through sEMG signals. Section III demonstrates the experimental results and the discussions. Section IV comes to the conclusions.

\section{MATERAILS AND METHOD}

\section{A. Apparatus}

To measure the surface EMG signal on the forearm, a 16 channel sEMG acquisition system is employed[18]. This device has a brand new electrodes array aiming to cover the most part of the muscle area on the forearm. 16 electrodes are fixed on a flexible sleeve with a certain pattern, as shown in Fig 1.

Comparing with traditional surface EMG technology that needs to stick pairs of electrodes on interested muscles, the system in this paper ignores the muscle positions. There are mainly 11 pieces of muscle in the forearm controlling finger and wrist motions according to anatomy. They are Extensor Carpi Uinaris (ECU), Extensor Digitorum (ED), Extensor Carpi Radiails Brevis(ECRB), Extensor Digiti Minimi (EDM), Flexor Carpi Ulnaris (FCU), Flexor Digitorum Profundus (FDP), Abductor Pollicis Longus (APL), Extensor Pollicis Brevis (EPB), Extensor Pollicis Longus (EPL) and Extensor Indicis (EE). All these muscles are close to their neighboring muscles, and some pieces of muscles even overlap the others. Therefore, it is not possible to pinpoint all

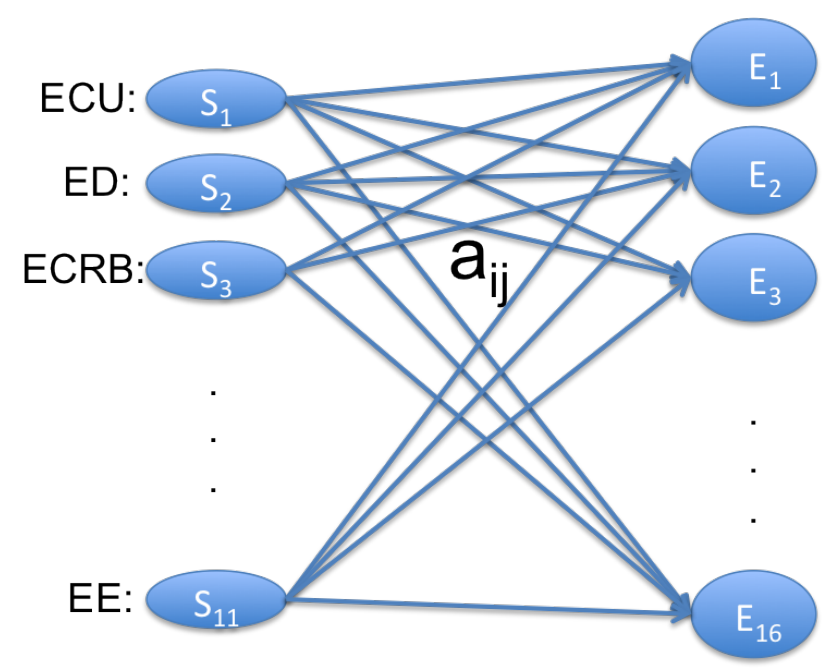

Fig. 2. The multi-input and multi-output system structure of 16 channel EMG acquisition system

the muscle and put corresponding EMG electrodes on them. Furthermore, crosstalk is a considerable issue in traditional sEMG technology as reported in [19]. To resolve these problems, a more general strategy is proposed by usig an specific electrodes configuration, which takes all muscles as the sources for each EMG channel, seen in Equation 1.

$$
E_{j}=\sum_{i=1}^{11} a_{i j} S_{i} \quad j=1 \cdots 16,
$$

where $E_{j}$ is the EMG signal measured from channel $j, S_{i}$ indicates the clean EMG signal of the $i^{\text {th }}$ muscle and $a_{i j}$ are the coefficients that demonstrate its weight to $E_{j}$ from muscle $i$. Figure 2 shows the signal composition of multimuscle sources.

To extract clean EMG signal from a noisy background, hardware is designed to amplify the EMG signal as well as remove various noises from power line interference, baseline wander, and so forth. The amplifier factor is 5000 with linear range $20 \mathrm{~Hz}$ to $500 \mathrm{~Hz}$. Differential mode amplifiers is utilised as the first-stage to remove the common mode noises, and then two Sallen-Key filters are employed to compose the band pass filter with a bandwidth between approximately 20 $\mathrm{Hz}$ to $500 \mathrm{~Hz}$. Furthermore, to suppress the power-line noise, a notch filter with central cut-off frequency at $50 \mathrm{~Hz}$ (UK power line frequency) is designed in hardware to remove power line noise.

Analog output from 16 EMG channels are connected to a micro-controller STM32L151V8 (STMicroelectronics Inc.), then digitised via a internal 12-bit analog-digital converter (ADC). The sampling frequency is $1000 \mathrm{~Hz}$.

FingerTPS system (Pressure Profile Systems, Inc. (PPS), USA) is utilised to capture the tactile force on the finger pulp with highly sensitive capacitive-based pressure sensors,seen in Figure 3. The sample frequency is $100 \mathrm{~Hz}$ controlled by the PC clock.

To synchronise the data of EMG system and FingerTPS 


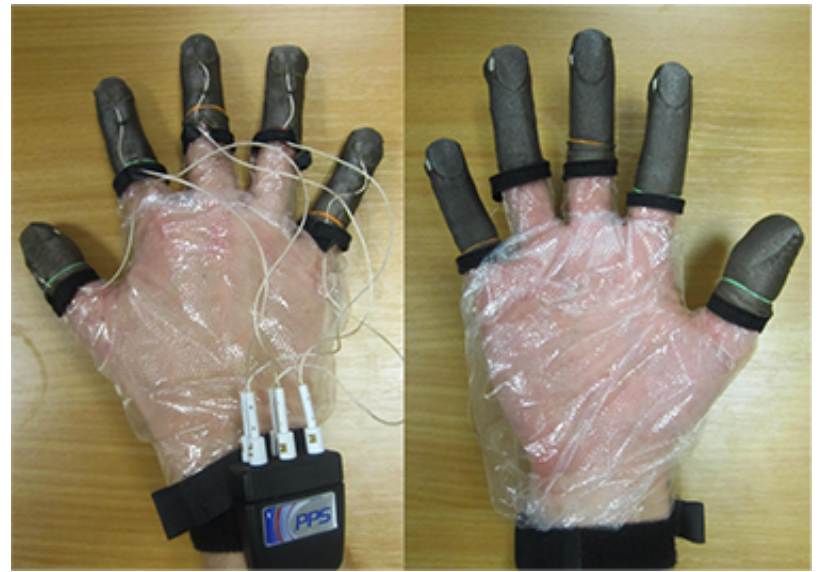

Fig. 3. The subject was asked to wear the FingerTPS sensors on each finger.

system, both devices are connected to a PC with Windows $\mathrm{XP}$ operating system via USB port, and multi-media timer is used to generate the synchronisation signal. An application based on MFC is specially designed to initialise two devices and record data. When capturing the data, subjects can observe the wave of signal on the screen.

\section{B. Subjects}

Three right-handed university staff ( 2 males and 1 female) served as subjects. [age: $30 \pm 6$ years, mass: $62.5 \pm 12.1$ $\mathrm{kg}$, height: $168 \pm 5.2 \mathrm{~cm}$ ]. The subjects had no previous history of neuropathies or traumas to the upper limbs. This project is approved by the ethic institution of university of portsmouth.

\section{Experimental procedure}

Subjects were required to wash their right forearm and hand using ordinary hand-wash liquid to remove horny and keeping forearm slightly moisture. Then the subjects were assisted by an expert to wear the electrodes sleeve in a similar approach, as seen in Figure 4.

The procedure are as follows. Firstly, make the palm upwards and identify the label line $b 1, b 2$ and $b 3$, which indicates the wrist angle, elbow and the middle line, respectively. Secondly, wear the sleeve from the end of End2 to End1, and pull the end of sleeve End2 to the line $b 2$ and End1 to $b 1$. Meanwhile, keep electrode 0 and electrode 12 on the middle line $b 3$. At last, wear another empty sleeve that has no electrodes to cover the previous sleeve for the sake of generating a squeeze to every electrodes towards the skin. It is suggested to wait 10 minutes to reduce the skin to electrodes resistance naturally.

Before wearing the FingerTPS sensor, subject need to wear a plastic glove in advance to isolate the electronic signal from FingerTPS sensor.

The subjects were seated in a chair beside the experiment desk facing the computer monitor with the upper arm on the armband, and the angle between the armband and the forearm is approximately $45^{\circ}$, as seen in Figure 5. The wrist and the

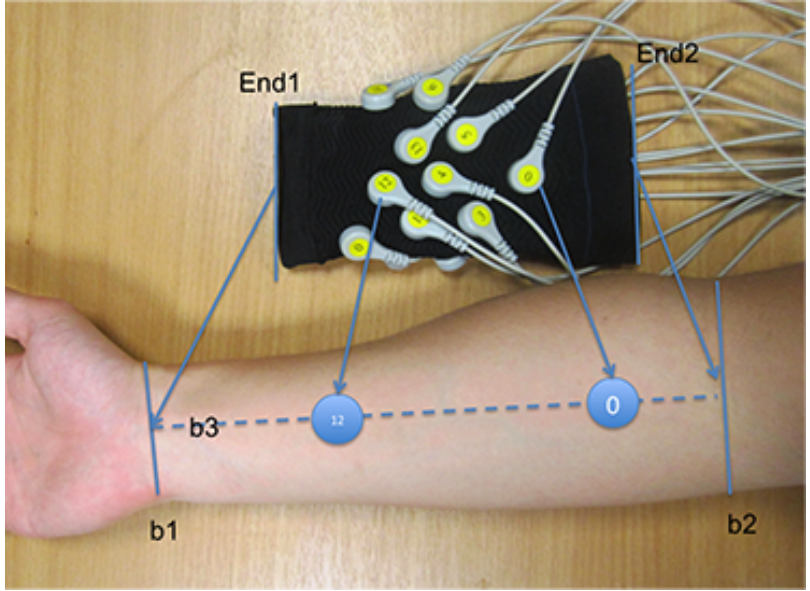

Fig. 4. This figure supplies the reference standard for wearing electrodes sleeve. End 1 and end2 are the two end of the sleeve. b1 and b2 indicate the positions of wrist and elbow, respectively. When the subjects wears the sleeve, End1 should be pulled to reach b1, and using End2 to reach b2.

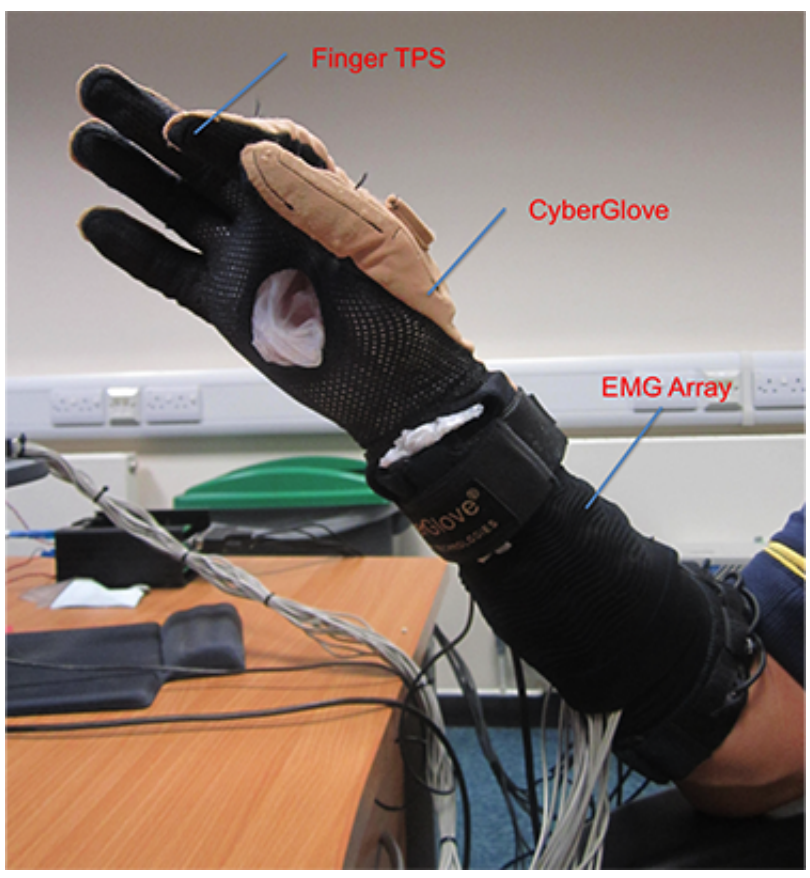

Fig. 5. The scene of capturing data from one subject

hand kept a relaxation state, which make the subjects feel the best comfortable. Then the subjects were asked to repeat four different finger gestures for 5 times, and between different gestures, 5 times fast fists were asked to divide different gestures. The gestures are using the thumb to touch other finger pulp, and then perform a graded exertion from $0 \%$ to $100 \%$ of the maximum voluntary contraction (MVC). The subjects can observe the force curve from the screen while exerting force on the finger, and they were told to make the curve as smooth as possible. A piece of data captured from subject one is demonstrated in Figure 7. 


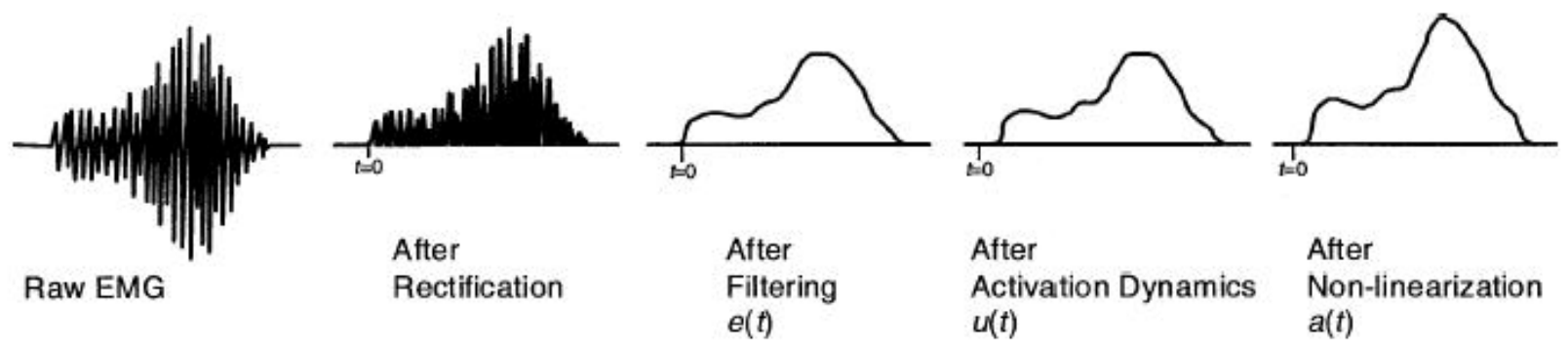

Fig. 6. Muscle activation dynamics: Transformation from EMG to muscle activation [8]

\section{EMG-driven Hill-type Models}

Many researchers who do large-scale neuromuscular modelling use Hill-type models, because these models are phenomenological in nature and less computationally timeconsuming [8]. The instantaneous total force, $F_{M T}$, exerted by a musculotendon unit is calculated by means of a lumped Hill-type musculotendon model [20], [21]

$$
F_{M T}(\theta, t)=\left(\widetilde{F L_{a}} \cdot \widetilde{F V} \cdot a(t)+F L_{p}\right) \cdot F_{o M}
$$

where $t$ is time, $\theta$ is the angular position of the figures. $\widehat{F L}_{a}(\theta, t)$ and $\widehat{F L_{p}}(\theta, t)$ are respectively the normalised active and passive muscle force-length relationships, $\widetilde{F V}(\theta, t)$ is the normalised muscle force-velocity relationship, $F_{o M}$ is the maximum isometric muscle force and $a(t)$ is the muscle activation[8].

This paper sets the angle of the joint $\theta$ to a constant, and thus the angular velocity is 0 , which leads to $\widetilde{F V}=$ 1 [20]. In addition, assume that the length of a muscle does not change while exerting force, thus $\widetilde{F L_{a}}(\theta, t)$ and $\widetilde{F L}_{p}(\theta, t)$ are constant as well, and the value of them can be referred in [20]. In addition, this paper assumes that all gestures are completed at the optimised muscular length, and therefore $\widetilde{F L}_{a}(\theta, t)$ is 1 and $F L_{p}(\theta, t)$ is 0 . As a result, the instantaneous total force $F_{M T}$ only depends on the muscle activation $a(t)$, as seen in Equation 3 .

$$
F_{M T}(t)=a(t) \cdot F_{o M}
$$

To obtain the $a(t)$ from raw EMG signal, the basic steps can be seen in Figure 6. In the first step, the raw EMG signal will be processed via normalisation, rectification and filter [8], [20], [21] to extract muscles activation profile, which is referred as $e(t)$ in this paper. Buchanan [8], etc., states EMG measuring the electrical activity that is spreading across the muscle, causing it to activate and induce the production of muscle force. It, however, takes time for the force to be generated. Thus there exists a time delay for the muscle activation. This process of transforming $e(t)$ to neural activation $u(t)$ is called Muscle Activation Dynamics, which has been approximately obtained by a discrete equation,

$$
u(t)=\alpha e(t-d)-\beta_{1} u(t-1)-\beta_{2} u(t-2)
$$

where $d$ is the electromechanical delay and, $\beta_{1}$ and $\beta_{2}$ are the coefficients that define the second-order dynamics with constraints of

$$
\begin{gathered}
\beta_{1}=\gamma_{1}+\gamma_{2} \\
\beta_{2}=\gamma_{1} \times \gamma_{2} \\
\left|\gamma_{1}\right|<1 \\
\left|\gamma_{2}\right|<1
\end{gathered}
$$

to guarantee its stability. In addition, to obtain a unit gain, the following equation must hold true.

$$
\alpha-\beta_{1}-\beta_{2}=1
$$

From neural activation $u(t)$ to muscle activation $a(t)$, it is a nonlinear map. This paper adopts the nonlinear relationship below to obtain $a(t)$

$$
a(t)=\frac{e^{A u(t)}-1}{e^{A}-1},
$$

where the constant $A$ is the only a parameter determining the nonlinear shape factor. It is allowed to vary between -3 and 0 in [8].

\section{E. Adjusting Parameters}

Studies of muscle force predictions usually compare muscle loading or activation patterns with EMG data as an estimate of validity. General analysis incorporate the quantification of muscle force sensitivity on modelling parameters and comparisons of muscle forces against direct measurements of tendon loading[5].

The model of estimating the forces of isometric contraction from EMG signal is described in the above section. In the process of gain $a(t)$ from $e(t)$, there are four coefficients are unknown, $d, \beta_{1}, \beta_{2}$ and $A$. $d$ indicates the electromechanical delay, which accounts for the delay between the neural signal and the start of the resulting twitch[8] and it has been reported to range from $10 \mathrm{~ms}$ to about $100 \mathrm{~ms}$. In equation 4, to guarantee stability, $-2<\beta_{1}<2,-1<\beta_{2}<1$. In addition, the nonlinear relationship constant $A$ is set to $-3<A<0$, as reported in [8], [21].

Genetic algorithm (GA) is utilised to optimise these four parameters through minimise the following objective function,

$$
J\left(d, \beta_{1}, \beta_{2}, A\right)=\frac{\sum_{t}(a(t)-F(t))^{2}}{\sum_{t}(a(t)-a \overline{(t)})^{2}},
$$

where $\mathrm{F}(\mathrm{t})$ is the loading force of the pinch gestures measured by the fingerTPS tactile sensor on thumb pulp. 


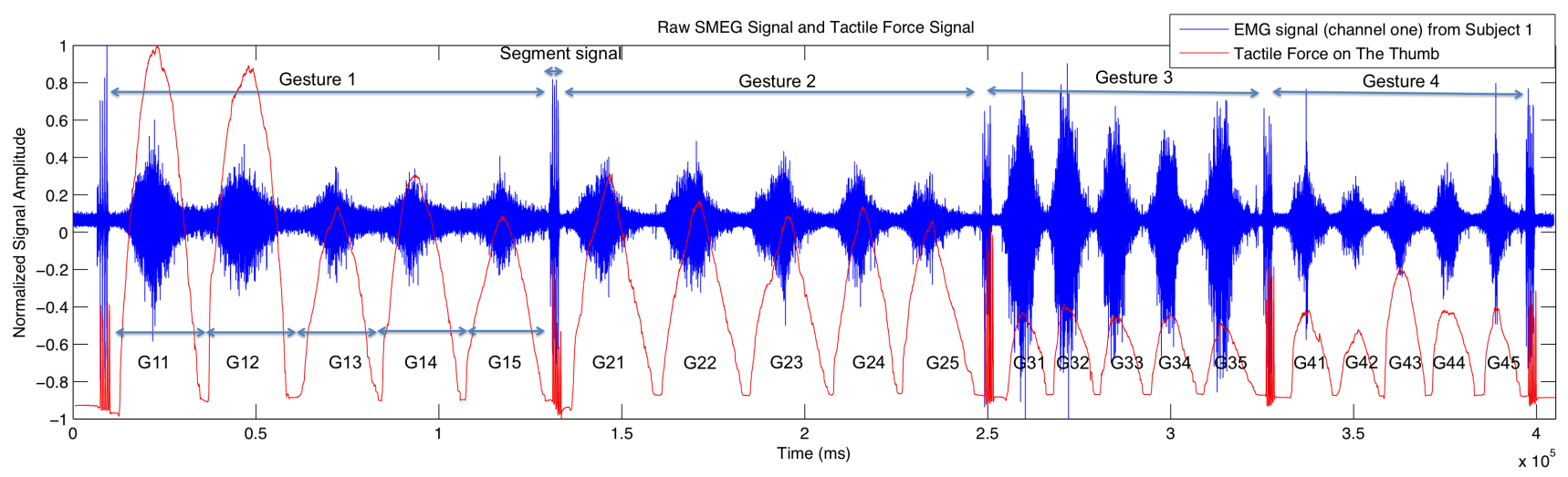

Fig. 7. This figure shows the data structure of raw sEMG signal and its tactile pinch force from subjects one. The signals are recorded continuously in a period of about 400 seconds. In this period, four types of gestures are performed, and each gesture is repeated five times. Between different gestures, five-times fist is used to separate each other. The blue wave in the figure is the raw EMG signal, and the red one is the pinch force. To show the EMG signal and force comparatively, both of them are normalised between -1 and 1 .

\section{RESUlTS AND DISCUSSIONS}

Figure 7 shows the raw sEMG signal (from sEMG channel 1, subject 1) and the pinch force. The pattern of pinch forces and EMG signal are rather complex. Because of some practical reasons, the MVC are not the same even for the same gestures. Consequently, the height of the force peaks varies as well as the amplitudes of sEMG signals. For instance, the force peak gesture G11 doubles that of G12. However, several reproducible phenomena can be identified. The pinch forces obtained from ring finger and little finger are smaller than that from the index finger and middle finger. The EMG signal of Gesture 3 is stronger than the other three. It is also easy to identify that when the sEMG signal reaches a peak, the force signal reach the peak as well. The overall trend of sEMG signal and force is consistent, and thus it is very likely to find the potential relationship between the EMG signal and the pinch force only through observing the raw data. Within this study we will concentrate on the interaction among the sEMG signal and the measured force during a single gesture.

Table I demonstrates the coefficient of determination $\left(r^{2}\right)$ using the processed sEMG signal to approach the measured force. Both single channel ( $\mathrm{C} 1$ to $\mathrm{C} 16)$ and a combination of the selected eight channels (Co) are used to estimate the force. The average $r^{2}$ of S1, S2 and S3 are 0.80, 0.71 and 0.77 , respectively. For $\mathrm{S} 1$, the preponderant half channels that can estimate the force better than the others are $\mathrm{C} 1, \mathrm{C} 3, \mathrm{C} 4$, $\mathrm{C} 9, \mathrm{C} 10, \mathrm{C} 11, \mathrm{C} 12, \mathrm{C} 13$, and these channels in S2 and S3 are $\mathrm{C} 1, \mathrm{C} 4, \mathrm{C} 5, \mathrm{C} 6, \mathrm{C} 7, \mathrm{C} 8, \mathrm{C} 11, \mathrm{C} 13$ and $\mathrm{C} 1, \mathrm{C} 2, \mathrm{C} 3, \mathrm{C} 4$, C5, C6, C8, C10, respectively. Among these channels, C1 and $\mathrm{C} 4$ appeared in all subjects, thus they can be considered as stable channels. Related analysis will be discussed later. Compared $r^{2}$ of the best single channel (It is C10 in G1, S1.) with $r^{2}$ of the combination channel $\mathrm{Co}$, the figures are rather close to each other with the deviation of $0.42 \% \pm 0.0317 \%$. Therefore, it can be concluded that if the best single channel can be found, the accuracy of force estimation utilising this single channel is still very high. However, it is not always the truth that the selected channel can keep stable in the whole precess of estimation. Thus, the next aim is to identify the tradeoff point between channel number and accuracy.

To identify the tradeoff point between the channel number and accuracy, 16 EMG channels were sorted with descending order according to the value of $r^{2}$ before selected. In this paper, the first one or several channels will be selected to form a new EMG signal, which ensure the channel with bigger $r^{2}$ will be selected first, and then the mean value of the selected multi-EMG will be calculated to form a new single EMG channel, seen in Equation 12.

$$
E M G(n, t)=\frac{1}{n} \times \sum_{i=1}^{n} E M G_{i}(t) \quad n=1 \cdots 16
$$

Figure 8, Figure 9 and Figure 10 demonstrate the changes of $r^{2}$ with the increase of the number of selected sEMG channels, respectively. For most gestures and subjects, it is easy to notice a trend that $r^{2}$ increases at the beginning (from 1 to 2 channels), then keeps stable from 2 to 6 channels, followed by a gradual decrease. So it is suggested to select 2 to 6 sEMG channels to estimate the force for a higher accuracy. The essential reason is that not all the muscles play the same role on a specific gesture. But the electrodes of the sEMG Sleeve cover all of them on the forearm. Some of them are master muscle and others are slaving muscles. Thus it is suggested to select the channels that cover the master muscles, then utilise these channels to estimate force. In addition, the fault tolerance of a system is related to the redundant information that the system can supply. The more EMG channel the system have, the more redundant the system will be. Therefore, using six channels to estimate force is more robust than using less channels.

\section{CONCLUSIONS}

This paper utilised the sEMG signal captured from a 16 channel SEMG Sleeve to estimate the pinch force of four gestures. Muscle activations were extracted to approach the force curve, and GA is used to optimise the coefficients. In this paper, three questions mentioned in Section I have 
TABLE I

REGRESSION ANALYSIS RESULTS OF DATA FOR MUSCLE ACTIVATION AS FORCE EXERTED BY EACH GESTURES WITH EXERTIONS RANGING FROM 0\% MVC TO $100 \%$ MVC OF THREE SUBJECTS.

\begin{tabular}{|c|c|c|c|c|c|c|c|c|c|c|c|c|c|c|c|c|c|c|}
\hline$r^{2}$ & Gs & $\mathrm{C} 1$ & $\mathrm{C} 2$ & $\mathrm{C} 3$ & $\mathrm{C} 4$ & C5 & C6 & C7 & $\mathrm{C} 8$ & $\mathrm{C} 9$ & $\mathrm{C} 10$ & $\mathrm{C} 11$ & $\mathrm{C} 12$ & $\mathrm{C} 13$ & $\mathrm{C} 14$ & C15 & $\mathrm{C} 16$ & $\mathrm{Co}$ \\
\hline \multirow{5}{*}{ S1 } & $\overline{\mathrm{G} 1}$ & .78 & .75 & $\begin{array}{l}.80 \\
\end{array}$ & 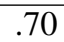 & .69 & 2.60 & .62 & .44 & .82 & $\mathbf{8 3}$ & $\mathbf{. 8 0}$ & $\frac{7.77}{27}$ & .77 & $\begin{array}{l}.33 \\
\end{array}$ & .08 & .63 & .83 \\
\hline & $\mathrm{G} 2$ & .82 & .69 & .81 & .76 & .78 & .70 & .57 & .18 & .75 & .72 & .70 & .70 & .79 & .58 & .41 & .68 & .79 \\
\hline & G3 & .88 & .85 & .90 & .79 & .64 & .49 & .61 & .69 & .88 & .87 & .85 & .78 & .71 & .01 & .01 & .74 & .89 \\
\hline & G4 & .64 & .37 & .59 & .53 & .57 & .63 & .58 & .40 & .63 & .55 & .55 & .52 & .61 & .32 & .33 & .50 & .67 \\
\hline & mean & .78 & .67 & .78 & .70 & .67 & .60 & .60 & .43 & .77 & .74 & .73 & .69 & .72 & .31 & .21 & .64 & .80 \\
\hline \multirow{5}{*}{ S2 } & G1 & .24 & - & $\begin{array}{l}.09 \\
\end{array}$ & .42 & .03 & .21 & - & .22 & - & - & .44 & .24 & .02 & .07 & - & .25 & .44 \\
\hline & G2 & .61 & .64 & .61 & .87 & .86 & .80 & .80 & .77 & .74 & - & .64 & .83 & .83 & .82 & .82 & .77 & .86 \\
\hline & G3 & .58 & .38 & .54 & .51 & .62 & .50 & .57 & .65 & .38 & - & .52 & .50 & .60 & .47 & .39 & .03 & .68 \\
\hline & G4 & .81 & .82 & .72 & .56 & .62 & .76 & .74 & .80 & .77 & .37 & .66 & .41 & .65 & .11 & .46 & .78 & .83 \\
\hline & mean & .56 & .46 & .49 & .60 & .54 & .57 & .53 & .61 & .48 & .09 & .57 & .50 & .53 & .37 & .42 & .46 & .71 \\
\hline \multirow{5}{*}{ S3 } & G1 & .55 & - & .45 & .68 & .65 & .53 & .20 & .42 & - & .79 & .20 & - & - & .07 & - & - & .81 \\
\hline & G2 & .73 & .76 & .78 & .80 & .82 & .74 & .72 & .77 & .68 & .78 & .22 & .77 & .67 & .78 & .56 & .39 & .83 \\
\hline & G3 & .81 & .72 & - & .60 & .34 & .10 & - & .01 & .61 & .43 & .56 & - & - & - & - & - & .80 \\
\hline & G4 & .56 & .60 & .51 & .56 & .18 & .42 & .46 & .56 & - & .61 & .19 & - & - & - & - & - & .62 \\
\hline & mean & .67 & .52 & .44 & .67 & .50 & .45 & .35 & .44 & .33 & .66 & .30 & .19 & .17 & .21 & .14 & .10 & .77 \\
\hline
\end{tabular}

Note: G1 is gesture 1 using index finger. G2 is gesture 2 using middle finger. G3 is gesture 3 using ring finger. G4 is gesture 4 using little finger. C1-C16 indicate the sEMG channel one to sEMG channel sixteen. Co indicates the combination of the best eight channels with the mean value of sEMG signal. The numbers in the table are the Coefficient of determination $r^{2}$. The symbol "-" in the table means the GA algorithm cannot reach an optimised output in a period of time, or the output is a negative.

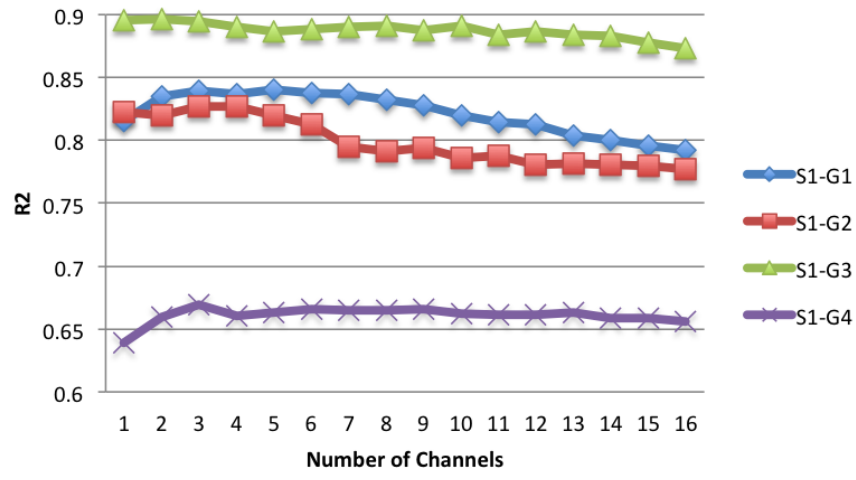

Fig. 8. The estimated forces change with different channel combination of subject 1. S1-G1 means Gesture one of first subject, and so forth.

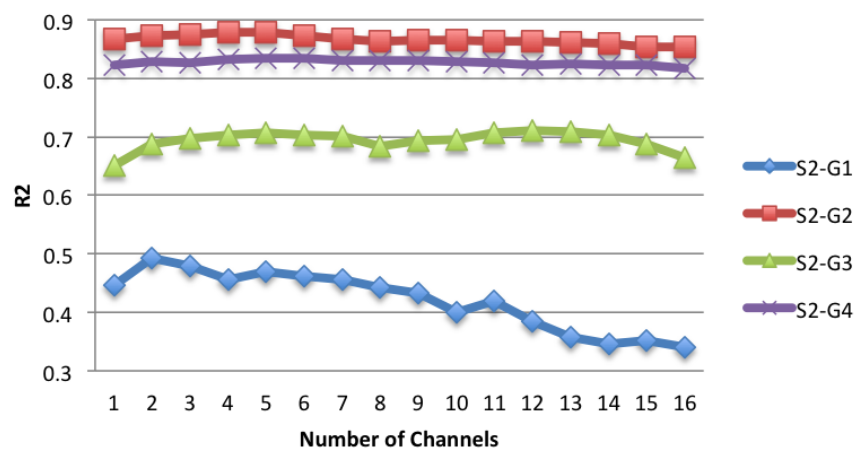

Fig. 9. The estimated forces change with different channel combination of subject 2. S2-G1 means Gesture one of first subject, and so forth.

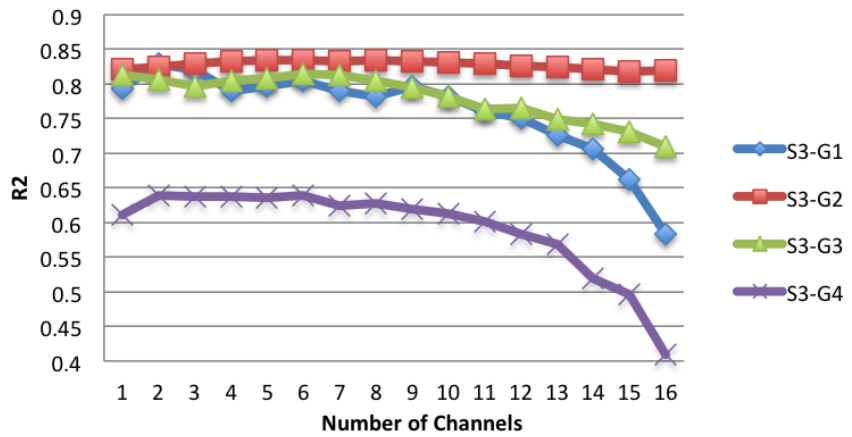

Fig. 10. The estimated forces change with the number of channel selected of subject 3. S3-G1 means Gesture one of first subject, and so forth.

been answered. Firstly, for a task of pure force estimation from sEMG signal (no physiological factors are considered ), it is practical to estimate the force only using muscular activations without muscular dynamic models. The process of muscle activation extraction can refer to Figure 6. Secondly, the results of this paper have shown that it is able to estimate the forces through the 16-channel sleeve regardless of muscle positioning on the forearm. Thirdly, this paper suggests to utilise six sEMG channels of he sEMG Sleeve to estimate the forces. These six channels are selected according to the capability of single channel performance, and the channels with high accuracy would be selected. Based on the above three points, a standard procedure for finger force estimation through sEMG sleeve can be concluded. The first step is performance evaluation for every single channel. Then, determine how many and which channels can be used 
to form a combination for every specific gesture. Finally, utilise the selected channel to evaluate the forces in practical applications.

\section{REFERENCES}

[1] Z. Ju and H. Liu, "Human hand motion analysis with multisensory information," IEEE/ASME Transactions on Mechatronics, vol. In press, 2013.

[2] Z. J. Ju and H. H. Liu, "Hand motion recognition via fuzzy active curve axis gaussian mixture models: A comparative study," Ieee International Conference on Fuzzy Systems (Fuzz 2011), pp. 699-705, 2011.

[3] Z. Ju and H. Liu, "Recognizing hand grasp and manipulation through empirical copula," International Journal of Social Robotics, vol. 2, no. 3, pp. 321-328, 2010.

[4] Z. J. Ju, G. X. Ouyang, M. Wilamowska-Korsak, and H. H. Liu, "Surface emg based hand manipulation identification via nonlinear feature extraction and classification," Ieee Sensors Journal, vol. 13, no. 9 , pp. 3302-3311, 2013.

[5] A. Erdemir, S. McLean, W. Herzog, and A. J. van den Bogert, "Modelbased estimation of muscle forces exerted during movements," Clinical Biomechanics, vol. 22, no. 2, pp. 131-154, 2007.

[6] M. Zecca, S. Micera, M. C. Carrozza, and P. Dario, "Control of multifunctional prosthetic hands by processing the electromyographic signal," Crit Rev Biomed Eng, vol. 30, no. 4-6, pp. 459-85, 2002.

[7] M. A. Oskoei and H. S. Hu, "Myoelectric control systems-a survey," Biomed Signal Process Control, vol. 2, no. 4, pp. 275-294, 2007.

[8] T. S. Buchanan, D. G. Lloyd, K. Manal, and T. F. Besier, "Neuromusculoskeletal modeling: Estimation of muscle forces and joint moments and movements from measurements of neural command," $J$ Appl Biomech, vol. 20, no. 4, pp. 367-395, 2004.

[9] M. Hayashibe, D. Guiraud, and P. Poignet, "Emg-based neuromuscular modeling with full physiological dynamics and its comparison with modified hill model," 2009 Annual International Conference of the Ieee Engineering in Medicine and Biology Society, Vols 1-20, pp. 6530-6533, 2009.

[10] F. M. Colacino, E. Rustighi, and B. R. Mace, "Subject-specific musculoskeletal parameters of wrist flexors and extensors estimated by an emg-driven musculoskeletal model," Med Eng Phys, vol. 34, no. 5, pp. 531-540, 2012.

[11] M. A. Maier and M. C. Heppreymond, "Emg activation patterns during force production in precision grip .1. contribution of 15 finger muscles to isometric force," Experimental Brain Research, vol. 103, no. 1, pp. 108-122, 1995.

[12] _ _ "Emg activation patterns during force production in precision grip .2. muscular synergies in the spatial and temporal domain," Experimental Brain Research, vol. 103, no. 1, pp. 123-136, 1995.

[13] E. J. Huesler, M. A. Maier, and M. C. Hepp-Reymond, "Emg activation patterns during force production in precision grip. iii. synchronisation of single motor units," Experimental Brain Research, vol. 134, no. 4, pp. $441-455,2000$.

[14] C. Choi, S. Kwon, W. Park, H. D. Lee, and J. Kim, "Real-time pinch force estimation by surface electromyography using an artificial neural network," Med Eng Phys, vol. 32, no. 5, pp. 429-436, 2010.

[15] B. J. Martin, T. J. Armstrong, J. A. Foulke, S. Natarajan, E. Klinenberg, E. Serina, and D. Rempel, "Keyboard reaction force and finger flexor electromyograms during computer keyboard work," Hum Factors, vol. 38, no. 4, pp. 654-664, 1996.

[16] V. M. Zatsiorsky, Z. M. Li, and M. L. Latash, "Enslaving effects in multi-finger force production," Experimental Brain Research, vol. 131, no. 2, pp. 187-195, 2000.

[17] _ - "Coordinated force production in multi-finger tasks: finger interaction and neural network modeling," Biological Cybernetics, vol. 79, no. 2, pp. 139-150, 1998.

[18] Y. Fang, X. Y. Zhu, and H. H. Liu, "Development of a surface emg acquisition system with novel electrodes configuration and signal representation," in 6th International Conference, ICIRA 2013, pp. 405414.

[19] J. P. M. Mogk and P. J. Keir, "Crosstalk in surface electromyography of the proximal forearm during gripping tasks," Journal of Electromyography and Kinesiology, vol. 13, no. 1, pp. 63-71, 2003.

[20] F. E. Zajac, "Muscle and tendon - properties, models, scaling, and application to biomechanics and motor control," Crit Rev Biomed Eng, vol. 17, no. 4, pp. 359-411, 1989.
[21] F. M. Colacino, E. Rustighi, and B. R. Mace, "An emg-driven musculoskeletal model for the estimation of biomechanical parameters of wrist flexors," 2010 Annual International Conference of the Ieee Engineering in Medicine and Biology Society (Embc), pp. 4870-4873, 2010. 